\title{
Reversal of Acquired Resistance to Doxorubicin in K562 Human Leukemia Cells by Astemizole
}

\author{
Masaaki IshiKaWa, ${ }^{*}$ Ryousuke FujIta, Motoaki Takayanagi, Yoshio TaKayanagi, and \\ Ken-ichi SASAKI \\ Department of Pharmacology and Toxicology, Cancer Research Institute, Tohoku Pharmaceutical University, 4-4-1 \\ Komatsushima, Aoba-ku, Sendai 981-8558, Japan. Received May 17, 1999; accepted October 5, 1999
}

\begin{abstract}
This study demonstrates that astemizole, a non-sedating anti-histaminergic drug with low toxicity in vivo, greatly potentiates the growth-inhibitory activity of doxorubicin in doxorubicin-resistant human leukemia cells (K562/DXR). Astemizole synergistically potentiated the cytotoxicity of doxorubicin for K562/DXR cells at a concentration of $0.1-3 \mu \mathrm{M}$ in a dose-dependent manner, whereas they showed hardly any synthergistic effect in the parental cell line (K562) at the same concentration. Since doxorubicin resistance in these cells is associated with the expression of high levels of P-glycoprotein, we evaluated the effect of astemizole on P-glycoprotein activity in cytofluorographic efflux experiments with doxorubicin. Our results indicate that astemizole inhibits the P-glycoprotein pump-efflux activity in a dose-related manner. Moreover, it also inhibits the photolabeling of P-glycoprotein by $\left[{ }^{3} \mathrm{H}\right]$ azidopine in a dose-dependent manner. These findings provide a biological basis for the potential therapeutic application of astemizole as an anticancer drug either alone or in combination with doxorubicin to multidrug-resistant leukemic cells.
\end{abstract}

Key words astemizole; doxorubicin; multidrug-resistance; human leukemia cell (K562)

Multidrug resistance (MDR) remains the major obstacle in the therapeutic cure of many malignances. A number of mechanisms of multidrug resistance at the level of plasma membrane, cytosol, and DNA levels, have been suggested. ${ }^{1-3)}$ The most common mechanism in the majority of MDR cell lines is an over expression of P-glycoprotein, a $170-\mathrm{kDa}$ metabolically active efflux pump glycoprotein. ${ }^{4)}$ Various agents, which were observed to attenuate resistance of tumors in animal studies, have been shown to inhibit the efflux of chemotherapeutics by competitively binding P-glycoprotein, thereby increasing the accumulation of chemotherapeutics in tumor cells. ${ }^{5,6)}$ One of the most typical agents is verapamil, a $\mathrm{Ca}^{2+}$ channel antagonist. ${ }^{7)}$ Clinical trials using verapamil showed successful results. However, a decrease in blood pressure and atrio-ventricular block in the heart were observed because verapamil exerts strong $\mathrm{Ca}^{2+}$ antagonistic effects on peripheral vessels and cardiac tissues. ${ }^{8)}$ Some investigators have shown that cyclosporin $\mathrm{A}$, a lipophilic agent that partitions between plasma membranes, reverses multidrug resistance both in vitro and in vivo. ${ }^{9,10)}$ Astemizole, a non-sedating anti-histaminergic drug that is metabolized by the cytochrome P-450 system (CYP 3A4), ${ }^{11)}$ has been shown to synergize with cyclosporin A. ${ }^{12)}$ Furthermore, an extensive literature review revealed a striking overlap between the substrates for and the inhibitors of both CYP 3A and P-glycoprotein. ${ }^{13,14)}$ Therefore, we set out to explore the possibility of whether similar synergism would exist between these two compounds in reversing MDR in vitro. As a part of the control experiment before doing experiments of synergism, we discovered that astermizole alone corrects intracullular drug accumulation and reverses drug resistance in human $\mathrm{K} 562 /$ DXR cells in vitro.

We used the human leukemic cell line K562, which dose not express P-glycoprotein, and its doxorubicin (DXR)-resistant variant K562/DXR, which expresses the MDR phenotype.

\section{MATERIALS AND METHODS}

Drugs and Reagents All chemicals were of analytical grade; doxorubicin chlorhydrate and rhodamine 123 were obtained from Sigma., St. Louis, MO, U.S.A. [ $\left.{ }^{3} \mathrm{H}\right]$ Azidopine was from Amersham., Arlington Heights, IL, U.S.A. Astemizole (Hismanal, Mochida Co., Ltd., Tokyo, Japan) was obtained commercially, and dissolved in dimethylsulfoxide (DMSO). Control cells were treated with the same amount of vehicle alone. The final DMSO concentrations never exceeded $0.5 \%(\mathrm{v} / \mathrm{v})$. Stock solutions of doxorubicin were made in distilled water and frozen at $-20^{\circ} \mathrm{C}$ until used. Light exposure was kept to a minimum for all drugs used.

Cell Lines and Culture A human leukemic cell line (K562) and a multidrug-resistant clone, K562/DXR derived from the K562 cell line were used. Properties of K562/DXR and the parental K562 clone have been described. ${ }^{15)}$ Cells were routinely kept in RPMI 1640 medium supplemented with $10 \%$ fetal calf serum and penicilline $\mathrm{G}(100 \mathrm{U} / \mathrm{ml}) /$ streptomycin $(100 \mu \mathrm{g} / \mathrm{ml})$ at $37^{\circ} \mathrm{C}$ in a humidified $5 \% \mathrm{CO}_{2}-$ $95 \%$ air incubator under standard conditions. Cell densities were determined by repeated cell counts using a hemato-cytometer, and cell viability was measured by counting the cells excluding those stained with $0.1 \%$ trypan blue. To maintain exponential growth, cells were seeded at $1 \times 10^{5}$ cells/ml and passaged every 4 to $5 \mathrm{~d}$.

Cytotoxicity Assay Cytotoxicity was measured by using a microculture tetrazolum (MTT) as described by Carmichael et al. ${ }^{15)}$ with modifications. Briefly, $1 \times 10^{4}$ cells were incubated in each well in a 96-well plates for $24 \mathrm{~h}$ prior to drug addition. Baseline sensitivities of the cells to astemizole alone were determined. Also, $25 \mu 1$ doxorubicin solution and $25 \mu \mathrm{l}$ astemizole solution diluted with medium were added.

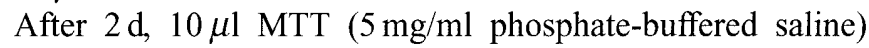
was added to each well, and the cells were incubated for $4 \mathrm{~h}$. Formazan crystals were dissolved by adding $100 \mu \mathrm{l}$ of DMSO after aspiration of the culture medium. The spectrophotometric absorbance at $570 \mathrm{~nm}$ was determined using a 
scanning multiwell spectrophotometer (Immuno Mini NJ2300 , Inter Med), and the cell surviving fraction values were calculated. Furthermore, the doxorubicin $\left.\mathrm{IC}_{50}{ }^{a}\right)$ values were determined in the presence of astemizole, while the $\mathrm{IC}_{50}{ }^{b)}$ obtained in the absence of astemizole, but in the presence of its solvent. The increased doxorubicin sensitivity or gain of sensitivity was calculated by the ratio: $\left.\mathrm{IC}_{50}{ }^{b}\right) / \mathrm{IC}_{50}{ }^{a)}$.

After treatment, cytotoxicity was determined by the trypan blue exclusion assay. Briefly, an aliquot of the cell suspension was diluted $1: 1(\mathrm{v} / \mathrm{v})$ with $0.4 \%$ trypan blue and the cells were counted with a hemocytometer. Results are expressed as the percentage of dead cells (ratio of stained cells $v s$. the control number of cells).

Cellular Accumulation of Doxorubicin To estimate doxorubicin in vitro, $1 \times 10^{6} \mathrm{cells} / \mathrm{ml}$ were resuspended in culture tubes in the presence or absence of various concentrations of astemizole. Doxorubicin $(4 \mu \mathrm{M})$ was added to the cells, gently mixed, and incubated at $37^{\circ} \mathrm{C}$. Tubes were removed at $45 \mathrm{~min}$ and cells for intracellular doxorubicin accumulation were analyzed by FACScan (Becton-Dickinson Co., San Jose, CA). Some 10000 cells were counted. All experiments were done in triplicate. Mean fluorescence was recorded from the histogram and data are expressed in the text as mean fluorescence channel numbers.

Binding of $\left[{ }^{3} \mathrm{H}\right]$ Azidopine to P-Glycoprotein Membrane vesicles from cells were prepared according the method of Sasa ${ }^{16)}$ except that cells were lysed using a Daunce homogenizer instead of nitrogen cavitation. The protein concentration of the vesicles was determined on $0.1 \%$ triton-solubilized samples using the BioRad protein assay. The ability of drug to displace labeled aszidopine from Pglycoprotein was assayed by a modification of the method of Sasa. ${ }^{16)}$ Drug binding to membrane vesicles was measured as follows: Plasma membranes ( $50 \mu \mathrm{g}$ protein) prepared from cells were photoaffinity labeled with $100 \mathrm{nmol}\left[{ }^{3} \mathrm{H}\right]$ azidopine (sp. act. $56 \mathrm{Ci} / \mathrm{mmol}$ ) in $40 \mathrm{~mm}$ Tris, pH 7.4, containing $4 \%$ DMSO in a final volume of $50 \mu \mathrm{l}$ in the absence or presence of 10 and $100 \mu \mathrm{M}$ astemizole. Photoaffinity-labeled membranes were solubilized in sodium dodecyl sulfate (SDS) and separated by electrophoresis in a $7.5 \% \mathrm{SDS} /$ polyacrylamide gel. The labeled bands were visualized by autoradiography.

\section{RESULTS}

Effect of Astemizole on Drug Sensitivity Drug sensitivity was measured by the MTT method colorimetric assay, a method which measures living cell content by virtue of mitochondorial activity as an indicator. The K562/DXR cell line had a relative resistance of about 600 -fold for doxorubicin, which represented the ratio of the doxorubicin $\mathrm{IC}_{50}$ of the K562/DXR cells to the doxorubicin $\mathrm{IC}_{50}$ of the $\mathrm{K} 562$ cells $(6.0 \mu \mathrm{M} / 0.01 \mu \mathrm{M}$; mean of 2 individual experiments). Therefore, a complete reversion of the resistance by a astemizole, as studied in Table 1, would consist in a 500-fold sensitization (gain of 500).

When astemizole was added at a final concentration of $0.1-3 \mu \mathrm{M}$ to the cells, astemizole potenciated the cytotoxicity of doxorubicin in a dose-related manner, and astemizole at $3 \mu \mathrm{M}$ completely reversed the doxorubicin resistance in K562/DXR cells; $2.6,3.5,7.5,66$ and 428 -fold sensitization was observed at $0.1,0.3,0.5 .1$ and $3 \mu \mathrm{M}$ astemizole, respec-
Table 1. Effect of Astemizole on $\mathrm{IC}_{50}$ of K562/DXR to Doxorubicin

\begin{tabular}{lcc}
\hline \hline Cell line & Astemizole $(\mu \mathrm{M})$ & Doxorubicin $\left(\mathrm{IC}_{50}: \mu \mathrm{M}\right)$ \\
\hline K562 & None & 0.012 \\
K562/DXR & None & 6.0 \\
K562/DXR & 0.1 & 2.3 \\
K562/DXR & 0.3 & 1.7 \\
K562/DXR & 0.5 & $0.8(3.5)$ \\
K562/DXR & 1 & $0.09(66)$ \\
K562/DXR & 3 & $0.014(428)$ \\
K562 & 3 & $0.01(1.2)$ \\
\hline
\end{tabular}

Cells $\left(1 \times 10^{4}\right.$ cells/well $)$ were incubated with various concentrations of doxorubicin in the absence or presence of five different concentrations of astemizole. After $48 \mathrm{~h}$ of continuous drug exposure, growth-inhibitory effects were evaluated by MTT assay. Each value represents the mean value for three different experiments performed in triplicate; the S.D. of the means were less than $10 \%$ and are omitted. Numbers in parentheses represent relative values as compared with the $\mathrm{IC}_{5 ! 1}$ obtained for corresponding cell line in the absence of astemizole.

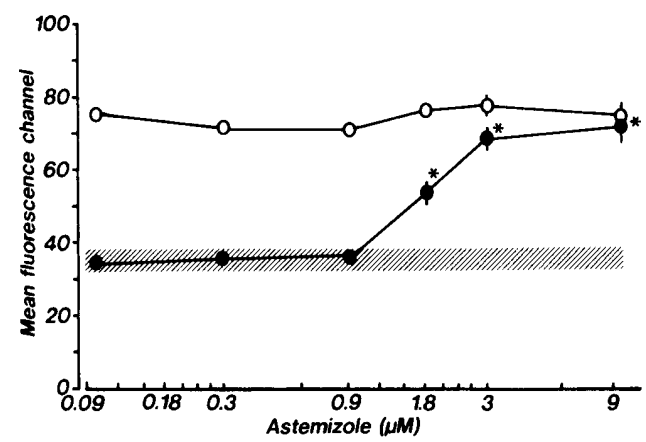

Fig. 1. Effect of Astemizole on Doxorubicin Accumulation in K562 and K562/DXR Cells

Cells ( $1 \times 10^{6}$ cells) were loaded with $4 \mu \mathrm{M}$ doxorubicin in the presence or absence of various concentrations of astemizole for $45 \mathrm{~min}$ and doxorubicin uptake measured, using FACScan in K562 (open circle) and in K562/DXR (closed circle). Data are expressed as mean fluorescence channel numbers; the S.D. of the means were less than $10 \%$ and are omitted. Dotted bar expresses mean \pm S.D. of K562/DXR cells alone. (* $p<0.05$ ) Significance of differences of values with astemizole from the K562/DXR cells.

tively. Astemizole itself ( $3 \mu \mathrm{M}$ ) had no cytotoxic effect to the K562/DXR cells. The effect of astemizole was approximately twice that of verapamil at 1 or $3 \mu \mathrm{M}$.; 30 and 165 -fold sensitization was observed at 1 and $3 \mu \mathrm{M}$ verapamil, respectively. But in $\mathrm{K} 562$ cells, astemizole at $3 \mu \mathrm{M}$ did not synergize the growth-inhibitory action of doxorubicin; at the concentrations examined and in the absence of doxorubicin, no cytotoxicity toward any K564 cell was detected by the trypan blue exclusion test. Astemizole circumvented resistance to vinblastine (VLB) in K562/VBL as well as K562/DXR cells (data not shown).

Effect of Astemizole on Intracellular Doxorubicin Accumulation to explore how astemizole potentiates the cytotoxicity of doxorubicin, the effects of astemizole on the accumulation of doxorubicin in K562/DXR was investigated. Cells were incubated with doxorubicin in the presence or absence of various concentrations of astemizole and intracellular accumulation was measured using FACScan. Doxorubicin efficiently accumulated in $\mathrm{K} 562$ cells at $37^{\circ} \mathrm{C}$, and astemizole and verapamil at $3 \mu \mathrm{M}$ did not affect this accumulation. In K562/DXR cells, the accumulation of doxorubicin was extremely reduced as compared to in K562-sensitive cells at $37^{\circ} \mathrm{C}$ (Fig. 1). Astemizole, in a dose-dependent manner, restored the doxorubicin accumulation in K562/DXR to a level comparable to that of K562 cells. Indeed, astemizole at $3 \mu \mathrm{M}$ 


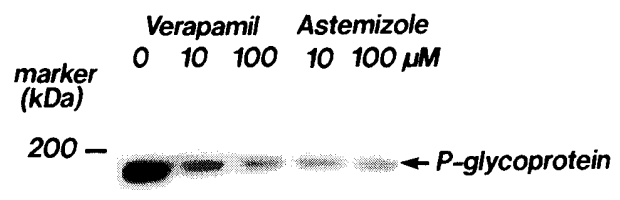

Fig. 2. The Inhibitory Effects of Astemizole on the $\left[{ }^{3} \mathrm{H}\right]$ Azidopine Photolabeling of P-Glycoprotein

Membrane vesicles ( $50 \mu \mathrm{g}$ of protein) from K562/DXR cells were incubated with a $\left[{ }^{3} \mathrm{H}\right]$ azidopine in the absence of DMSO, verapamil or astemizole as described in Materials and Methods. After solubilization, photolabeled proteins were fractionated on sodium SDS polyacrylamide gel electrophoresis and visualized by autoradiography. Typical photolabeling patterns are presented. Another experiment produced the same pattern.

enhanced the accumulation of doxorubicin in K562/DXR cells to an extent almost comparable with that observed in parental K562 cells incubated without astermisole. Verapamil at $3 \mu \mathrm{m}$ caused moderate enhancement of doxorubicin accumulation in K562/DXR cells. When the cells were incubated at $0{ }^{\circ} \mathrm{C}$ to suppress the intracellular energy system, no potentiation of doxorubicin accumulation by either astemizole or verapamil was observed (data not shown).

Inhibition of $\left[{ }^{3} \mathbf{H}\right]$ Azidopine Photolabeling of P-Glycoprotein by Astemizole To study the interactions of P-glycoprotein with astemizole, the effect of the binding of $\left[{ }^{3} \mathrm{H}\right]$ azidopine to membrane vesicles from K562/DXR cells was examined. Azidopine, a photoactive analog of dihydropyridine, photolabels P-glycoprotein in plasma membranes of MDR cells; this labeling is inhibited by vinblastine and some calcium channel blockers. ${ }^{17,18)}$ Tsuruo et al. have reported that a photoactive analog of verapamil photolabels P-glycoprotein in the plasma membranes of K562/DXR. ${ }^{9)}$ Using this photolabeling system, we investigated whether astemizole inhibited the $\left[{ }^{3} \mathrm{H}\right]$-azidopine photolabeling of $\mathrm{P}$ glycoprotein. As shown in Fig. 2, $\left[{ }^{3} \mathrm{H}\right]$ azidopine specifically labeled a 170000 - to 180000 -Da protein in K562/DXR cells but not in drug-sensitive K562 cells. The photolabeling of Pglycoprotein in K562/DXR membranes by $\left[{ }^{3} \mathrm{H}\right]$ azidopine was inhibited by astemizole in a concentration-dependent manner, and astemizole at $10 \mu \mathrm{m}$ partially, and at $100 \mu \mathrm{M}$ strongly inhibited the P-glycoprotein labeling. Verapamil at 10 and $100 \mu \mathrm{M}$ also partially inhibited the P-glycoprotein labeling, but its inhibition was less than astemizole. This result suggests that astemizole directly interacts with P-glycoprotein and inhibits the transport of doxorubicin.

\section{DISCUSSION}

The development of acquired MDR is a major obstacle to the chemotherapeutic cure of cancer. A number of mechanisms have been proposed for MDR. ${ }^{1-3)}$ Several studies have shown an association between MDR and decreased accumulation and enhanced efflux of drug, and over expression of Pglycoprotein, a metabolic active efflux pump. ${ }^{1,2)}$ In addition, increased levels of glutathione, glutatione S-transferase, and topoisomerase II have been documented as mechanisms for MDR. ${ }^{2)}$ It is apparent that different mechanisms for MDR are operational in different tumor cell lines. It is also likely that there are mechanisms for MDR yet to be discovered.
In this study, we examined the reversing effects of astemizole on multi-drug resistance because anthracyclines such as doxorubicin are known to bind P-glycoprotein, and agents capable of binding P-glycoprotein often show chemotherapeutic effects on neoplastic tissues or reverse multidrug resistance. It was found that astemizole exerted reversing effects on multidrug resistance in vitro. The degree of the reversing potency was stronger than that of verapamil in vitro.

The mechanism of action of astemizole appears to be mediated through inhibition of P-glycoprotein. K562/DXR and K562/VLB cells are known to express P-glycoprotein, but K562 cells do not. ${ }^{15)}$ These results suggest that astemizole is effective against P-glycoprotein-positive tumors. The ability of astemizole to increase the accumulation of doxorubicin in K562/DXR cells (Fig. 1) and rhodamine 123 in K562/DXR cells, and to block the efflux of rhodamine 123 (data not shown), a compound known to be a substrate for the putative drug-transporter, ${ }^{19)}$ supports the conclusion that astermizole acts by interfering with a process associated with the expression of P-glycoprotein. Although astermizole increased doxorubicin accumulation by 2 - to 3 -fold, it sensitized K562/DXR cells by 2 - to 420 -fold. This disproportion between drug accumulation and cytotoxicity is frequently observed ${ }^{20)}$ but remains poorly understood.

Because of compelling evidence in support of a specific action of astemizole on P-glycoprotein, the results of displacement of $\left[{ }^{3} \mathrm{H}\right]$ azidopine from P-glycoprotein were somewhat unexpected but not without precedent. For example, the concentration of drug such as vinblastine or doxorubicin required to displace azidopine is often a 2000-fold excess over the photoaffinity probe, ${ }^{17)}$ and certain drugs known to be affected by P-glycoprotein, such as colchicine, do not alter the photoaffinity labeling of the protein. ${ }^{21)}$ There are several explanations for these results. For example, astemizole may act at a site near but not identical to that of azidopine on P-glycoprotein, or it may produce its effect by several mechanisms including inhibiting enzymes having a putative role in modulating the function of P-glycprotein such as protein kinase C. ${ }^{22-24)}$ These alternatives are currently under investigation.

Although many drugs have been shown to sensitize MDR cells, most have proven to be unacceptably toxic in the clinic. ${ }^{8)}$ Astemizole, at usual doses, is relatively non-toxic. Not only did it lack the usual sedating effects of other antihistaminergic drugs, it has been safely administered to animals in doses of over $100 \mathrm{mg} / \mathrm{kg}^{25)}$ However, case reports have appeared in which patients overdosing on astemizole demonstrated prolongation of the QTc interval during cardiac monitoring, ${ }^{7)}$ indicating that changes in cardiac conduction could become problematic when the drug is used at higher doses. In vivo, astemizole is rapidly converted to a desmethylastemizole metabolite so that the concentration of the parent drug is virtually undetectable in the serum. ${ }^{25)}$ The activity of the metabolites against MDR cells should also be investigated since if they lose their activity, the usefulness of astemizole in vivo could be limited.

The identification of astemizole as a potent modulator of MDR is important; 1) astemizole represents a new class of clinically available drugs that could be exploited for use in patients harboring malignancies that express P-glycoprotein and display the MDR phenotype, and 2) it will be possible to search for more potent and effective derivatives for future 
evaluation in the clinic since many cogenerators of astermizole have been synthesized and are available for study.

In conclusion, as a new multidrug resistance modifier, astemizole showed strong resistance-reversing activity by a mechanism similar to that of verapamil; astemizole increases intracellular concentrations of chemotherapeutic agents by inhibiting the function of P-glycoprotein. These properties make astemizole a candidate for multidrug resistance modifiers.

\section{REFERENCES}

1) Moscow J. A., Cowan K. H., J. Natl. Cancer Inst., 80, 14-20 (1988).

2) Hayes J. D., Wolf C. R., Biochem. J., 272, 281-295 (1990).

3) Arceci R. J., Blood, 81, 2215-2222 (1993).

4) Jurank P. F., Zastawny R. L., Ling V., FASEB (Fed. Am. Soc. Exp. Biol.) J., 3, 2583-2592 (1998).

5) Cornwell M. M., Pasten I., Gottesman M. M., J. Biol. Chem., 262 , 2166-2170 (1987).

6) Akiyama S., Cornwell M. M., Kuwano M., Pasten I., Gottesman M. M., Mol. Pharmacol., 33, 144-47(1988).

7) Tsuruo T., lida H., Tsukagoshi S., Sakurai Y., Cancer Res., 41, $1967-$ 1972 (1981)

8) Dalton W. S., Grogan T. M., Meltzer P. S., Sheper R. J., Duie B. G., Taylor C. W., Miller T. P., Salmon S. E., J. Clin. Oncol., 7, 415-24 (1989).

9) Slater L. M., Sweet M., Stupecky M., Gupta S., Br. J. Cancer., 54, $235-239$ (1996).
10) Slater L. M., Sweet M., Stupecky M., Gupta S. C., J. Clin. Invest., 77, $1405-1408$ (1996).

11) Goss J. E., Ramo B. W., Blake K., Arch. Intern. Med., 153, 27052712 (1993).

12) Tren D., Brett W., Jahnchen E., Birnbaum D., Lancet, ii, 1335-1336 (1987).

13) Zhou X.-J., Zou-Pan X.-R., Gauthier T., Placidi M., Maurel P., Rahmani R., Biochem. Pharmacol., 45, 853-861(1993).

14) Zhou-Pan X.-R., Sérée E., Zhou X.-J., Placidi M., Maurel P., Barra Y., Rahmani R., Caner Res., 53, $5121-5126$ (1993).

15) Sasaki T., Ishikawa M., Kannno S., Takayanagi M., Takayanagi Y., Sasaki K., Res. on Drug Action Interac., 22, 41 -51 (1998).

16) Sasa A. R., Proc. Natl. Acad. Sci. U.S.A., 85, 7187-7191(1988).

17) Sasa A. R., Glover C. J., Meyers M. B., Biedler J. L., Felsted R. L., J. Biol. Chem., 262, 7884-7888 (1987).

18) Tang C.-P. H., Mellado W., Horwitz W., Biochem. Pharmacol., 37, 1417-1421(1988).

19) Tapero H., Munck J.-N., Fourcade A., Lapidis T. J., Cancer Res., 44, 5544-5549 (1984).

20) Ford J. M., Hait W. N., Pharmacol. Rev., 42, 155-199 (1990).

21) Cornwell M. M., Sasa A. R., Felsted. R. L., Gottesman M. M., Pasten I., Proc. Natl. Acad. Sci. U.S.A., 83, 3847-3850 (1986).

22) Palayoor S. T., Stein J. M., Hait W. N., Biochem. Biophys. Res. Commun., 148, 718-725 (1987).

23) Fine R.L., Patel J., Chabner B. A., Proc. Natl. Acad. Sci. U.S.A., 85, $582-586$ (1988).

24) Chambers T. C., McAvoy E. M., Jacobs J. W., Eilon G., J. Biol. Chem., 365, $7679-7686$ (1990).

25) Davies A. J., Harinda V., McEwan A., Ghose R. R., Br. Med., 298, 325 (1989). 